\title{
Next steps in implementing Kaput's research programme
}

\author{
Celia Hoyles \& Richard Noss \\ Institute of Education, University of London
}

\section{Abstract}

We explore some key constructs and research themes initiated by Jim Kaput, and attempt to illuminate them further with reference to our own research. These 'design principles' focus on the evolution of digital representations since the early nineties, and we attempt to take forward our collective understanding of the cognitive and cultural affordances they offer. There are two main organising ideas for the paper. The first centres around Kaput's notion of outsourcing of processing power, and explores the implications of this for mathematical learning. We argue that a key component for design is to create visible, transparent views of outsourcing, a transparency without which there may be as many pitfalls as opportunities for mathematical learning. The second organising idea is that of communication, a key notion for Kaput, and the importance of designing for communication in ways that recognise the mutual influence of tools for communication and for mathematical expression.

\section{Introduction}

In an extraordinarily comprehensive and challenging review of technology and mathematics education, Jim Kaput (1992) mapped out a framework for understanding the roles of technology in the field, with a particular focus on the theme that would occupy his attention for the rest of his life - the evolution of notational systems for expressing mathematics. In this paper, we have used several of Kaput's major papers as 'data' from which to draw out a set of related ideas that can justifiably be called 'Kaput's principles'. These principles are grounded in Kaput's research; we aim to rehearse the arguments and extend them where we can in order to contribute to a 
coordinated research agenda that will situate Kaput's work in the broader field, and seek to take it forward.

\section{Background}

It is now 40 years since the first email. In that time, communication between individuals has been transformed, not only in terms of how we communicate, but what might be said and to whom. It has created (difficult, in English at least, to avoid the technocentric formulation that suggests that the technology is the primary agent) new forms of technical infrastructure - like threading and smart mailboxes - and these, in turn, have helped to transform the technology into an invisible part of the culture most of us routinely leave the $e$ off email.

Yet email has had no proximal effect on our major concern, that is mathematical learning. Why should it? Would we expect any technological change to have an effect (however defined) on a particular aspect of learning? Surely not: it would be strange indeed if each piece of a technology itself had a specific and measurable outcome on some educationally relevant practice. Of course, it has had a distal effect: teachers communicate with each other via email - they, like everyone else, have come to think of communication as instantaneous, global. In the sense that the social and professional lives of teachers and researchers have been transformed, email has had its effect. But this is not a proximal effect - there is, to the best of our knowledge, no attempt to research the possibilities of email for the mathematics classroom. (Maybe there should be: we do, after all, have evidence that communicating mathematical ideas to a distant and invisible peer can encourage a formal mode of discourse between students: Noss et al., 2002).

One issue that naturally arises, therefore, is to ask what are the necessary conditions for a technology radically to shape transformational change in the learning and teaching of mathematics (it is, of course, clear that finding sufficient conditions, though desirable, is unachievable)? This is an important problem for mathematics education, as it encourages us to look not only at what is special about technologies, but what is special about mathematics to be learned. It is a structural, epistemological 
problem, concerning knowledge transformation, as well as what might be functionally possible to learn. It is a problem that was at the heart of so much of Jim Kaput's research work. In his seminal paper, Kaput (1992) argued:

\begin{abstract}
Anyone who presumes to describe the roles of technology in mathematics education faces challenges akin to describing a newly active volcano - the mathematical mountain is changing before our eyes, with myriad forces on it and within it simultaneously. Many of these forces have a technological component ... the same technological forces that shape the mathematics also deeply affect the teachability and learnability of mathematics, both old and new' (p. 515).
\end{abstract}

Trying to understand how technology can shape (and be shaped by) mathematical knowledge and its expression has kept us occupied for much of this time, a preoccupation about the interrelationship between technology and epistemology that we shared with Kaput. A major concern for us both has been to describe how mathematical abstraction is scaffolded within computational media, and to seek legitimacy for alternative representational systems that harness them. Our way of thinking about this problem has centred around understanding how mathematical expression can at once appear as decontextualised and yet - notably in the context of suitably-crafted technology - remain connected to and situated in the linguistic and conceptual web of resources afforded by the medium and the activity system (see Noss \& Hoyles, 1996; Hoyles, Noss and Kent, 2004, for a discussion of situated abstraction and ongoing theoretical work by, for example, Lobato (2005) who brings the notion of agency into the theoretical discussion).

When we recently undertook a review of technology and mathematics over a decade after Kaput's 1992 paper, we were again faced with the enormity of the volcano, and its extreme level of activity. Like Kaput, we noted that the incorporation of technologies into mathematical learning almost inevitably brings to the fore a range of key questions - particularly those concerned with transformation of the what of mathematics rather than merely the how - precisely because digital technologies disrupt many taken-for-granted aspects of what it means to think, explain and prove mathematically and to express relationships in different ways (Hoyles \& Noss, 2003). In the remainder of this paper, therefore, we will seek to elaborate some of these ideas 
by focusing on what we distinguish as four design principles generated by Kaput's work.

\section{First principle: attend to representational infrastructure}

In his 1992 paper, Kaput starts with a comparison between our current relationship with computers and that of owners of the Model $\mathrm{T}$ Ford, a car for which a rather intimate knowledge of its functioning was necessary before it could be driven. Kaput points out that in 1992 we were computationally at the point of the 'latter days' of the Model A., in which necessary intelligence needed to operate the car had 'migrated' from person to machine, standardisation had emerged, and previous modes of transport were beginning to fade:

\footnotetext{
We have progressed beyond the Model T, where one needed to be an expert to start and run the vehicle, where standards for automobile operation were not yet established, and where competition with older modes of transportation were important factors. (p. 516).
}

Perhaps so, although struggling with the idiosyncrasies of the word processor we are using, it sometimes doesn't feel quite like that, even now! In any case, it is important to appreciate that the evolution of the technology of the car - how it came to be that expertise for driving a car became straightforward and non-expert - is not only a story of technology enhancement. It is a story of infrastructural transformation as well. As Kaput put it:

... rules of the road gradually came to be standardized and codified as experience dictated needs and redefined solutions. ... one needed a protocol to deal with frightened and startled horses, for example. .. And, of course, the roads themselves, reasonably well suited for earlier modes of transportation, needed rebuilding to accommodate the requirements of automobiles, which could not approach their real potential on roads not designed for them. (p. 517).

It is worth exploring Kaput's point a little more. We would not ask, now at least, whether a car would arrive at its destination sooner than a horse drawn vehicle - but we could have asked once. What has changed? Partly the possible speed of the car. But without suitable roads and rules by which we drive on them, no amount of 
technological enhancement would improve journey time - in some conditions, a horse drawn vehicle would certainly arrive faster. The key design principle we derive from Kaput's analogy is that the car, the necessary infrastructure, and rules of discourse that governed their interaction co-evolved simultaneously, forming a triad that creates what he described as a representational infrastructure ${ }^{1}$. Once defined, Kaput pointed to the need for mathematics educators to attend to this infrastructure because of its influence not only on how mathematics is learned but what is learned. We noted (Kaput, Hoyles \& Noss, 2002) that although general questions of representational infrastructures may seem somewhat remote from the task of learning mathematics, it raises to the fore the central challenge of designing learnable systems. Such systems depend on the representional forms with which they are expressed, shaping and sometimes defining what can be considered as learnable. The difficulty of a mathematical idea often inheres in the system with which it is expressed - imagine just how difficult it would be to remember the various procedural rules of calculus (like the chain rule) without Leibniz's elegant notation.

A similar argument is proposed by Papert (2006), who invites us to join him in a thought experiment at the time when Roman numerals were in use:

Imagine that the small number of people capable of doing multiplication was an obstacle to economic progress and that the learning scientists were funded to mobilize all the great ideas in How People Learn to remedy the situation. Undoubtedly better teaching would increase the number of people capable of performing the complex art of multiplication. But something else did this far more effectively: the invention of Arabic arithmetic turned an esoteric skill into one of "the basics". (p. 582).

This observation is by no means trivial, not least because of the invisible - or at least taken-for-granted - character of the mathematical infrastructure, a point to which we shall return later. The point is that some infrastructural changes are so powerful, so transformative in, not just the possibilities of new pedagogy, but of epistemology they redefine what counts as possible, what is expressible. The principle is clear: the

\footnotetext{
${ }^{1}$ As a thought experiment, it is interesting to imagine what could have developed as a standard car if the job of transforming the terrain proved simply too intractable: A very different kind of vehicle would have evolved, and very different kinds of social relations to maintain its function.
} 
infrastructure, and in particular the relations between its constitutive elements, are legitimate objects of study, crucial to understanding what mathematics might be expressed and how, and who is capable of success. We only remark here that our own work on this issue has taken two distinct forms: first, the possibilities, in designing new kinds of representational infrastructures, of opening new trajectories for learners who cannot adequately deal with conventional representational forms; and second, in studying the roles of the invisible mathematics of workplaces, and how these can be explicitly addressed. We elaborate both of these issues below.

\section{Second principle: work for infrastructural change}

We now turn to consider the second principle, perhaps more aptly seen as a corollary of the first: the potential of new representational infrastructures to facilitate change in what is learned and who can learn it, to explore how far we have progressed from the model T. In a paper we prepared together (Kaput, Hoyles \& Noss, 2002), we noted how little impact digital technologies had been made on mathematics teaching and learning:

\footnotetext{
The appearances of new computational forms and literacies are pervading the social and economic lives of individuals and nations alike. Yet nowhere is this upheaval correspondingly represented in educational systems, in classrooms, or in school curricula. As far as mathematics is concerned, the massive changes to mathematics that characterize the late twentieth century - in terms of the way it is done, and what counts as mathematics - are almost invisible in the classrooms of our schools and, to only a slightly lesser extent, our universities. (pp. 51).
}

We suspect that the situation remains rather similar even in these early years of the twenty-first century. Kaput's abiding focus was on the hegemonic role of algebraic expression as a means to express mathematical structure in pedagogical settings. Indeed, it is clear how algebra often serves as a barrier to students being able to articulate generality (see Küchemann and Hoyles, in press) even though the need to express and justify generality is "the heart, root and purpose of algebra" (Mason, 2005 , p. 2). This difficulty - essentially with the pedagogic appropriateness of the representational infrastructure as a means for novices to express mathematical ideas has been well documented and theorised (see, for example, Bednarz et al. 1996, Healy 
\& Hoyles, 2000, Stacey et al, 2004). Moreover, Küchemann and Hoyles (in press), also showed that although the use of structural reasoning increases with age among high-attaining students aged 13-15 years (that is, reasoning by reference to infrastructure in whatever mode of expression) it does so only at a very modest rate alongside widespread and largely inappropriate pattern spotting or calculation.

In a forthcoming edited volume, Algebra in the Early Grades, Kaput and colleagues (Kaput, Carraher \& Blanton, in press) brought a new dimension to our understanding of these results by locating the problem in the historical roots of the "elitist" mathematics curriculum, taking account of whom, and for what purpose, mathematical expression was historically considered appropriate (interestingly, this theme is echoed in Papert, 2006, who uses this argument to refute the Chomskian claim that human evolution has occurred in ways that are incompatible to algebraic thinking). Kaput et al. identified a range of representational innovations, all of which required a computational medium for their realization but did not require algebraic infrastructure for their use and comprehension, thus gaining increased accessibility for more learners. But of course, not every computational medium would fit this agenda; a key property Kaput identified was that phenomena were at the centre of the representation experience, so learners could see the results of their actions on representations of the phenomenon, and vice versa.

While mathematisation of phenomena is undoubtedly a powerful criterion for classifying transformative potential, the mechanism for achieving mathematisation is no less important. We argued recently, in a review of research concerning mathematics and digital technologies, (Hoyles \& Noss, 2003), that we should seek to consider not only the ways in which digital technology shape mathematical learning through novel infrastructures, but also how it is shaped by its incorporation into mathematical learning and teaching contexts. Furthermore, apart from its unsurprising dependence on tasks, pedagogical interventions and activity structures, our review of research suggested that learning using potentially transformative digital technologies was highly sensitive to small changes in the technologies, and the micro-structures of infrastructure. So we would include an addendum to this second principle: the necessity to include detailed activities and their design criteria alongside any agenda of infrastructural change. To return to the driving metaphor, we must attend to the 
quality of the car, the road and the need for rules and regulations to fit with the goals of car users: where people want to go and how.

In seeking to characterise key components of new representational infrastructures, Kaput (1992) focused on the potential of dynamic media to link actions, noting that 'cognitions that also monitor connections between representations are quite unlikely cognitive resources are exhausted in carrying out the translations'. (p. 530). He also suggested that it might not be 'linked representations' that are the crucial means to effect these translations, but rather the affordance of the representational infrastructure to point, link or trigger another system. This is, of course, a critical capacity of mathematical thought: mathematical objects acquire their conceptual power from the ability of the thinker to conjure up properties, internal and external relationships, theorems and - last, and in meaning-generation terms, least definitions. One possible design corollary (there are many) is to design environments in which a common representational infrastructure links diverse representational forms, by, for example, a programming language (see, for example, diSessa 2000).

Of course, this last strategy begs several questions, not least about the status of such a representation, whether it somewhow stands above the representations to which it is linked, or is better considered as an instance of a class of representations none of which have privileged semiotic (or epistemological) significance. Neither should we underestimate just how problematic is the conceptual linkage of disparate representations for the student (see for example, Duval, 2000). Nontheless, in a paper some ten years ago, we worked on just this problem of common representational infrastructure that we briefly revist below (Noss, Healy \& Hoyles, 1997).

In a microworld Mathsticks, we linked direct manipulation of computational objects with the description of their arrangement in sequences as Logo programs. Our intention was to help students build links between seeing, doing and expressing through the common representational infrastructure of Logo, to investigate how mathematical meanings were structured by the tools available for expressing the relationships under study and to trace how far this mediational process assists in the construction of the mathematical meanings intended by their teachers or curriculum designers. 
Mathsticks is an instance of a class software that we termed autoexpressive: that is, a virtual world in which action within the environment entails and depends upon linguistic expression of the mathematical objects ${ }^{2}$. The key idea is that fuzzy intuitions concerning relationships are simultaneously expressed through and elaborated within a linguistic framework. Our attempt was to construct a language that was simultaneously rigorous and expressive, mathematically precise and learnable (see, for example, Sherin, 2001).

Neither the details of the Mathsticks environment, nor the degree of success we achieved are especially pertinent. The idea of autoexpression tried to capture something special about digital technologies, or rather something special about the design of software that might encourage the development of mathematical meaning. By privileging the role of language, we aimed to express our conviction that linguistic representation, in the form of textual - and in this case, programmable - code, leverages a kind of expressive power, that could act as a replacement - in learning for traditional algebraic expression (see Sacristán \& Noss, in preparation, for a more recent example on the same theme).

With hindsight, while maintaining the view that expressing relationships and coming to see the implications and limitations of one's expression, is a crucial role for microworlds we now recognise that textual expression is an instance of a more general class in which explicit representation - with or without text - is the key element. It is explicitness that is key, as it directly addresses the problem of the invisibility of infrastructure we referred to earlier. Our research with several nontextual representational systems (see e.g. Hoyles \& Noss, 2003; Simpson, Noss \& Hoyles 2005) has nevertheless raised difficult questions, not least an appreciation both of the potential of dynamic graphical, animated infrastructures but the unparalleled opportunity afforded by text as an object on which to reflect - either by individual learners or groups.

\footnotetext{
${ }^{2}$ We are grateful to David Shaffer for helping us to elaborate this idea of autoexpression (personal communication: see also, Shaffer, 2005).
} 
Additionally, we were confronted with the issue of how non-textual representations can be aligned with standard mathematical notation and more fundamentally the problem that, as Kaput noted, school mathematics is all too often only about these notations:

\footnotetext{
Virtually every historically received formal system in the school mathematics curriculum evolved as a representation system ... They were thus primarily aimed at developing student competence in the syntax of some formal system. pp. 545-6.
}

Here is, in our view, the key reason why alternative infrastructural solutions (Logo, Boxer, etc.) have yet to impact fundamentally on learning in mathematics. Put bluntly, attempts to replace one representational system with another strike at the very heart of what the mathematics educational enterprise believes it is about: the notation system is the territory not just the map ${ }^{3}$ so that replacing it with another however well-tuned it may be epistemologically, and however learnable it may be remains problematic.

\section{Third principle: outsource processing to the computer but attend to the implications}

Kaput's basic argument, elaborated in many papers since 1992, is that human history is entering a fifth phase, a virtual culture based on the externalisation of symbolic processing. The outsourcing of processing power from the human mind to the machine is the critical contribution that technology will potentially make, not least because of the opportunity to create "democratizing infrastructures which will redefine school knowledge" (Kaput, Hoyles \& Noss, 2002).

Perhaps the most straightforward research issue, in the sense of symbols coming alive to be processed externally, is to ask what kinds of roles external symbolic processing play in the generation of mathematical meaning. Obvious exemplars of external processing are computer algebra systems, such as Mathematica. The potential of such

\footnotetext{
${ }^{3}$ Wikipedia helpfully reveals that Alfred Korzybski's most famous saying means that "an abstraction derived from something, or a reaction to it, is not the thing itself".
} 
systems, as Kaput puts it, is that students can come to see mathematics as a 'fundamental way of making sense of the world, reserving most exact computation and formal proof for those who will need those specialized skills' (Shaffer \& Kaput, 1999). Extraordinary possibilities emerge, including - to coin a phrase - a New Kind of Science (Wolfram 2002), a science that would be simply impossible with predigital technologies.

Given the remarkable potential for epistemological and pedagogical innovation that such systems embody, it is not surprising that attention has recently focused on the implications of this outsourcing trend for what new knowledge might be acquired and by whom (Noss \& Hoyles, 2006). This work has centred on illustrating how mathematical learning is crucially linked, not only to the actions and attitudes of the teacher but on how far the students master what the French school of researchers term 'the process of instrumentation': the extent to which the learner is aware of the system, and is able to look through it as well as look at it (Artigue 2002). This strand of work entails a more sensitive realisation that there is a fine balance between the 'pragmatic' and 'theoretical' (or 'epistemic') roles of calculation.

We have argued elsewhere (Hoyles, Noss and Kent, 2004), that the instrumental genetic analysis of students' evolving mathematical knowledge leaves relatively unexplored the texture of situated abstractions of mathematical ideas that are being developed and expressed, and how these abstractions are webbed by the available tools and shaped by the interactions with these tools and with the community. This is a critical point, since although schemes of instrumented action supply a powerful tool for conceptualising the process of tool-learner interaction, there is considerably more that needs to be done in terms of the kinds of mathematical knowledge that develop in such interactions. Primarily, this knowledge, or rather the ways in which it is expressed, may not look or sound like standard mathematical discourse: indeed, if the representational system underpinning the tool is non-standard, it follows that the knowledge will be similarly non-standard. This is what the notion of situated abstraction seeks to address: it allows us to recognise and legitimate mathematical expression even when it is remote from (or not represented by) standard mathematical discourse. The notion is particularly salient in computational environments, since it is the nature of interactive, dynamic representations that they encourage expression (and 
therefore, initially at least, conceptualisation) that diverges from standard mathematics. Unless this is recognised, the genuinely transformative potential of digital technology representations will remain unacknowledged (see Noss \& Hoyles, 1996).

Returning to the problem of outsourcing, the possibility of simply leaving to the machine the tedious and fragile business of arithmetic or algebraic calculation is, from a mathematical perspective truly momentous, and from a pedagogical perspective, clearly advantageous - but not unambiguously so. If the role of the calculation is merely to achieve an answer, then outsourcing it to a machine is unproblematic. But if, as the French workers have shown, there are aspects of calculation that also contribute to meaning-making, then the wholesale removal of technical expertise may have some unanticipated effects on the perception of mathematics as a coherent whole (see also, Goldenberg 2000).

In this paper, we take a further look at the problem of outsourcing in an unusual but telling context - that of the workplace. Workplaces are distinguished from classrooms in many ways, one of which is particularly salient - there is (mostly) no explicit intention either to teach or learn, and especially not to teach or learn mathematics. Mathematics is invisible, even in workplaces that are avid consumers of mathematical knowledge in the form of computational systems (see, for example, Kent \& Noss, 2000). In such cases, the outsourcing of processing power reaches extreme limits (at first sight, it often looks as though no mathematical knowledge is required by operators of computational systems).

We have been studying the construed meanings of symbolic artefacts in workplaces in our project Techno-mathematical Literacies (TmL) at work ${ }^{4}$. By techno-mathematics, we mean the mathematics embedded in (and invisible within) technological artefacts; and by literacies, we signal our intention to focus on the interpretation and functional

\footnotetext{
4 The Techno-mathematical Literacies in the workplace project [http://www.ioe.ac.uk/tlrp/technomaths] isfunded by the Teaching and Learning Research Programme [http://www.tlrp.org], a programme of the U.K. Economic and Social Research Council (Award no. L139-25-0119).
} 
use of mathematical relationships rather than any explicit production of mathematical relationships (see, for example, Hoyles \& Noss, in press; Hoyles, Bakker, Kent \& Noss, in press; Noss, Bakker Hoyles \& Kent, 2007).

Much of our work has focused on studying just what kind of TmL are tacitly or explicitly employed in technology-rich workplaces or are needed for more effective work. But we have recently turned out attention to systematic attempts to foster TmL, and to make transparent, where possible and appropriate, the mathematical relationships that underpin the workplace knowledge in use much of which has been outsourced to the machine. We take as a starting point symbolic artefacts that are familiar in the workplace and are intended to serve as boundary objects for communication between sub-communities in the workplace or between employees and their customers (Kent et al. in press).

Let us take as an example, our work in the retail financial sector, a company we will call "Lifetime Pensions". We encountered a ubiquitous artefact, the "annual pension statement". The statement aimed to allow customers to share information held by the company on the state of their pension investment. All the calculations embodied in the statement were, of course, outsourced to a sophisticated computer system.

We found that typically, customers would contact the company unable to make sense of the statement, or querying details within it. This necessitated explanations from the "Enquiry Team", often augmented by texts prepared by technical experts, sent (only) to the team. However, we found that most of the members of the Enquiry Team perceived the workings of all the underlying models as equally opaque, while in fact some were rather trivial (lump sum calculations), while others were extremely technical (annuity calculations). To summarise, we concluded that the accumulation of automated systems over time, had resulted in knowledge impoverishment of employees in two distinct but related ways. First, the systems separated employees from any appreciation of the relevant simple models and calculations, and rendered them unable to communicate their structure and implications to colleagues and customers. An evocative example is that in a group of eight employees in Lifetime pensions, we found none who had ever considered that there existed any relationship between monthly and annual interest rates - other than company convention! Second, 
the system had rendered almost all the employees unable to distinguish information derived from simple models from those that could be answered only by adapting an existing solution by reference to technical experts.

Our response to this challenge was to identify symbolic artefacts in the workplace as potential boundary objects to co-design with employees, computer tools that allowed reconstructions of these artefacts in a layered way: that is so that some of their underlying mechanisms could be made progressively accessible, either by offering access to engage with the structure of the financial instrument or by providing visually intuitive feedback of the effects of different input variables on the model (Bakker et al. 2006). In essence, our approach was to take the pension statement and recast it so that the shaping of the mathematical relationships were foregrounded. We used two different technological infrastructures, one utlising spreadsheets where employees were invited to express their understandings of the statement in terms of the 'language' of the spreadsheet and to generalise this over several time periods or across different instruments using compound interest. Second, we designed a standalone web-based tool with which employees could explore the interactions between time, interest rate, and frequency of payments. We also designed activities, often based on scenarios we has observed and found a readiness - even enthusiasm on the part of many employees and trainers to work with us. We encountered first surprise that any relationships existed, let alone mathematical relationships that could actually be expressed by employees, some of whom had limited mathematical background, but later a growing confidence (one employee noted that "it was not as difficult as I thought!).

What insights did we gather in relation to outsourcing? The assumption that all processing and calculation of mathematics can be embedded in a computer system, and thus be of concern only to technical specialists, clearly leads to huge difficulties in communication between different groups. We propose that the superficial similarity of mathematical knowledge at school and at work, (take, for example, the idea of compound interest) masks a significant distinction. The 'effect' of workplace context is to introduce a significant degree of complexity to even the simplest mathematics. Any mathematical procedure is not an isolated exercise but is part of a set of decisions 
and judgements that have to be made about what is a complex process or product. In Lifetime Pensions, the actuarial assistant phrased it thus:

"The maths involved is not hard, but it is applied in a very complicated way - there are the company rules and Inland Revenue [tax] rules". It is this complexity that is generally invisible and needs to be articulated so that in some cases at least, the calculations will become less problematic".

The mathematics buried in this complexity is - due to the outsourcing of processing to the system - invisible; indeed, it often appears not to be mathematics at all. And so an unexpected result of devolving processing to computers, at least in the workplace, is that mathematical meaning becomes harder - not easier - to construct. The removal of the need to calculate does not - as we might have expected - provide the chance to focus on the structures, but rather removes from sight the structures themselves.

It is this challenge that arises, albeit in a different form, in schools too. Our wellintentioned attempts to outsource processing to computational systems - results in parts of the mathematical system being obscured, even as others are revealed. We have shown the potential of a layered approach. It is here that we might gain some leverage by exploiting the idea of autoexpression, as a means to reconnect epistemic knowledge with pragmatic: there is, after all, a substantial difference between using a machine in which calculation has been outsourced, and expressing the means by which - say, in the form of an algorithm - the outsourcing should occur. Exploring the theoretical implications of this should, we argue, form part of any future research agenda.

\section{Fourth principle: exploit connectivity to encourage sharing and discussion}

We now turn to a fourth and final principle enunciated by Kaput, and one to which we can only give cursory attention. In his most recent work, Kaput argued that the connectivity made possible by computational media constituted a profoundly important set of affordances, ranking alongside the 'representational-simulation affordances' of computational media. He argued that the advent of broadband and low-cost (especially handheld) technology in classrooms offered considerable potential although he noted that, like any other technology, it could only reach fruition if it was integrated into classroom practice. Kaput was realistic about the research 
challenges involved, noting that "present uses of such technology are either representationally sophisticated but connectivity-limited (e.g. our prior SimCalc work) or connectivity sophisticated but representationally impoverished (e.g. commercially available ClassTalk systems).” (Roschelle \& Kaput, 2005; p. 6).

Our own response to this challenge has been to connect representational innovation in the WebLabs ${ }^{5}$ project, seeking to provide an autoexpressive medium with which to explore mathematical problems and to model solutions again without text and algebra (Noss \& Hoyles, 2006; Simpson et al., 2005, 2006) and also to devise means by which students could share and critique each others' models across the internet. We achieved this by building sets of open tools that could be combined to construct more complex functionalities; these were built in ToonTalk (Kahn 1996), a state-of-the-art programming system where abstract computational concepts are represented by concrete animated analogues. Alongside this, we devised a web-based system, WebReports, that allowed students to post their ideas - and their working models and to interpret and reflect upon them in collaboration with their peers, some of whom might be working in other classrooms. (see http://www.lkl.ac.uk/kscope/weblabs).

In practice, however, this last step proved to be much more difficult than we imagined. Many of the difficulties were pragmatic but more fundamentally, the tools for communication and the tools for the construction of the models were to some extent not sufficiently aligned. We did not manage sufficiently to develop the students' sense of ownership of inter-site group reports, and a common understanding of their purpose. In fact, we came to recognise the critical role for an inter-site "motivator" and are currently investigating the possibilities for automating this role, which might be the first step in introducing some intelligent support using the latest innovations in technology and connectivity to fostering collaboration. (ref)

We did however, achieve remarkable success in encouraging discussion through our WebReports of mathematical ideas in which the interchange was based around

5 Grant IST 2001-3220 of the Information Society Technologies Programme of the European Commission. We acknowledge the contribution of all the WebLabs team, and notably the UK researchers, Y. Mor and G. Simpson. See http://www.weblabs.eu.com. 
students challenging each other's models competitively rather than building a joint outcome. For example, one challenge was to find the rule underlying a sequence followed by the quest for more elegant solutions (Mor, Noss, Hoyles \& Simpson, 2004), another to guess the movement of a moving car represented by a particular velocity-time graph. These interchanges were sustained over several weeks with increasingly sophisticated explanations invoked. In short, we found that the tools and activities, and the new representational and communicative infrastructure - each of which had been iteratively designed by us for more than two years - were far from sufficient. Our most successful activities stemmed from the emergence of a cultural infrastructure, that arose alongside the technical and pedagogic It is much too soon to predict how the infrastructural relationships will play themselves out in the presence of highly connected technologies: some very promising technical approaches are already mature (see e.g. Wilensky, 1999). But it is not unlikely that the outsourcing of connection - i.e. designing the technology to construct some of the cultural norms of the classroom in place of human interaction, will bring with it similar problems to those already experienced by the outsourcing of calculation.

\section{Concluding remarks}

Kaput's vision has begun to bear fruit, with more attention being directed at alternative representational systems, and a more explicit focus on the design of communication-rich settings. At the same time, as the outsourcing of calculations, processing and now connectivity to machines becomes the norm, the strengths and limitations of outsourcing are becoming clarified, and a viable research programme to assess learning potential is becoming defined.

Our interest, like Kaput's, has been to understand what makes digital media qualitatively different from other technologies that have promised so much, and yet have delivered so little (Shaffer and Kaput, 1999), at least in the classroom. Thanks to Kaput, the common over-optimistic trajectory for research in the use of digital technologies for innovative mathematical change has been curtailed: there has been early recognition of the cycle of over-enthusiasm and disillusion and a more ready appreciation of the need for a more nuanced documentation of potentials and 
In Educational Studies in Mathematics, 68. 2, 85-94 Page 18 of 21

obstacles, appreciation of tool mediation, tasks and activities, and the acknowledgment of the teacher's role. Key challenges remain: above all, we have argued, to introduce transparency into the outsourcing of processing power and to find ways to support this process and learning more generally, through innovative development of learning communities undertaken by teachers and - perhaps the time is finally here - by machines.

\section{References}

Artigue, M. (2002). Learning Mathematics in a CAS environment: The genesis of a reflection about instrumentation and the dialectics between technical and conceptual work. International Journal of Computers for Mathematical Learning, 7, 3, 245-274

Bakker, A., Hoyles, C., Kent, P., \& Noss, R. (2006). Improving work processes by making the invisible visible. Journal of Education and Work, 19, 4, 343-361.

Bednarz, N., Kieran, C., \& Lee, L. (Eds.). (1996). Approaches to algebra: Perspectives for research and teaching. Dordrecht, The Netherlands: Kluwer.

diSessa, A. (2000). Changing Minds, Computers, Lesarning And Literacy. Cambridge, MA: MIT Press.

Duval R. (2000) Basic issues for research in mathematics education. In: Nakahara, T. \& Koyama M. (Eds). Proceedings of the $24^{\text {th }}$ Conference of the International Group for the Psychology of Mathematics Education. Hiroshima Univeristy 1, 55-69

Goldenberg P. (2000) Thinking (and talking) about technology in Math Classrooms. Education Development Center. http://www2.edc.org/mcc/PDF/iss_tech.pdf

Healy, L. \& Hoyles, C. (2000), 'A Study of Proof conceptions in Algebra'. Journal for Research in Mathematics Education, 31, 4, 396-428.

Hoyles, C. and Noss, R. (2003) 'What can digital technologies take from and bring to research in mathematics education?' In A.J. Bishop, M.A. Clements, C. Keitel, J. Kilpatrick and F.K.S. Leung (eds), Second International Handbook of Mathematics Education. Dordrecht: Kluwer Academic Publishers.

Hoyles, C., and Noss, R. (in press). The meanings of statistical variation in the context of work. in Lesh, R., Hamilton, E. \& Kaput, J. (Eds.) Mathematical 
In Educational Studies in Mathematics, 68. 2, 85-94 Page 19 of 21

Abilities needed for Success beyond School in the 21st Century. Hillsdale, NJ: Lawrence Erlbaum Associates.

Hoyles, C., Bakker, A., Kent, P., \& Noss, R. (in press). Attributing meanings to representations of data: The case of statistical process control. To appear in Mathematical Thinking and Learning.

Hoyles, C., Bakker, A., Kent, P., \& Noss, R. (in press). Attributing meanings to representations of data: The case of statistical process control. To appear in Mathematical Thinking and Learning.

Hoyles, C., Noss, R. \& Kent, P (2004) On the Integration of Digital Technologies into Mathematics Classrooms. International Journal of Computers for Mathematical Learning, 9, 3, 309-326.

Hoyles, C., Noss, R. and Kent, P. (2004), 'On the Integration of Digital Technologies into Mathematics Classrooms'. International Journal for Computers in Mathematical Learning., 9, 3, 309-326

Kahn, K. (1996). ToonTalk - An animated programming environment for children. Journal of Visual Languages and Computing 7, 2, 197-217.

Kaput, Carraher \& Blanton, (in press) Algebra in the Early Grades

Kaput, J. (1992) Technology and Mathematics education. In D. Grouws (ed.), Handbook of Research on Mathematics Teaching and Learning, pp. 515-556. New York: Macmillan.

Kaput, J., Hoyles, C. \& Noss, R. (2002) Developing New Notations for a Learnable Mathematics in the Computational Era. In English, L. (Ed) Handbook of International Research in Mathematics Education. London: Lawrence Erlbaum. pp. 51-75.

Kent, P., Noss, R., Guile, D., Hoyles, C., \& Bakker, A (in press). Characterising the use of mathematical knowledge in boundary crossing situations at work. To appear in Mind, Culture, and Activity, special issue on "Learning and Technology at Work".

Kent, P. \& Noss, R. (2000) The visibility of models: using technology as a bridge between mathematics and engineering. International Journal of Mathematics Education in Science and Technology. 31, 1, 61-69.

Küchemann, D \& Hoyles C. (in press) From empirical to structural reasoning in mathematics: tracking changes over time in Blanton,M., Stylianou,D. and 
In Educational Studies in Mathematics, 68. 2, 85-94 Page 20 of 21

Knuth, E (Eds) The Learning and Teaching of Proof Across the Grades Lawrence Erlbaum

Lobato, J. (2005, April). The actor-oriented abstraction approach: Coordinating individual and social levels of abstraction. In J. Lobato (Chair), Abstraction in mathematics learning: Comparing alternative emerging conceptions. Symposium conducted at the annual meeting of the American Educational Research Association, Montréal, Canada

Mason, J. (2005) Frameworks For Learning, Teaching And Research: theory and practice, Plenary presentation, in Wilson S. et al (Eds) Frameworks That Support Research \& Learning: Proceedings of PME-NA.

Mor, Y., Noss, R., Hoyles C. \& Simpson, G. (2006) Designing to see and share structure in number sequences. International Journal for Technology in Mathematics Education. 13 (2), 65-78.

Noss, R. \& Hoyles, C. (1996) Windows on Mathematical Meanings: Learning Cultures and Computers. Dordrecht: Kluwer.

Noss, R. \& Hoyles, C. (2006) Exploring Mathematics through Construction and Collaboration. In K.R. Sawyer (Ed) Cambridge handbook of the Learning Sciences. Cambridge: CUP. pp. 389-405.

Noss, R., Bakker, A., Hoyles, C., \& Kent, P. (2007). Situating graphs as workplace knowledge. Educational Studies in Mathematics. Vol 65, (3), 367 - 384

Noss, R., Healy, L. and Hoyles, C. (1997). The construction of mathematical meanings: Connecting the visual with the symbolic. Educational Studies in Mathematics, 33, 2, 203-233.

Noss, R., Hoyles, C, Gurtner, J-L., Adamson, R. \& Lowe, S. (2002) Face-to-face and online collaboration: appreciating rules and adding complexity. International Journal of Continuing Engineering Education and Lifelong Learning 12, 5/6, $521-539$

Papert, S. (2006) Afterward: After How Comes What. In K.R. Sawyer (Ed) Cambridge handbook of the Learning Sciences. pp. 581-586. Cambridge: CUP. Roschelle, J. \& Kaput, J. (2005) Understanding Math Classroom Affordances of Networked, Hand-Held Devices. Unpublished proposal.

Sacristan, A. \& Noss, R. (in preparation) Computational Construction as a Means to coordinate representations of infinity. 
Shaffer, D. W. (2005). Studio mathematics: The epistemology and practice of design pedagogy as a model for mathematics learning (WCER Working Paper No. 2005-3). Madison: University of Wisconsin-Madison, Wisconsin Center for Education Research. http://www.wcer.wisc.edu/publications/workingPapers/

Shaffer, D.W. and Kaput, J. (1999). Mathematics and virtual culture: a cognitive evolutionary perspective on technology and mathematics education. Educational Studies in Mathematics, 37, 2, 97-119

Sherin, B. (2001) A comparison of programming languages and algebraic notation as expressive languages for physics. International Journal of Computers for Mathematics Learning. 6, 1-61

Simpson, G. Hoyles, C. \& Noss, R. (2006) Exploring the mathematics of motion through construction and collaboration. Journal of Computer Assisted Learning. 22, pp.1-23, 2007

Simpson, G., Hoyles, C. \& Noss, R. (2005) Designing a programming-based approach for modelling scientific phenomena. Journal of Computer Assisted Learning. 21, pp. 143 - 158.

Stacey, K., Chick, H., \& Kendal M. (2004) The Future of the Teaching and Learning of Algebra. Dordrecht: Kluwer.

Wilensky, U., NetLogo [Computer software]. 1999, Center for Connected Learning and Computer-Based Modeling: Evanston, IL.

Wolfram, S. (2002) A New Kind of Science, Champaign: Wolfram Media Inc. 